\title{
Subserous Type of Eosinophilic Colitis: A Rare Disease
}

\author{
Carolina Amado ${ }^{1}$, Mariana Silva Leal $^{1}$, Pedro Neto $^{1}$, Gisela Ferreira $^{2}$ \\ ${ }^{1}$ Internal Medicine Department, Centro Hospitalar do Baixo Vouga, Aveiro, Portugal \\ ${ }^{2}$ Haematology Department, Centro Hospitalar do Baixo Vouga, Aveiro, Portugal
}

Received: 26/05/2021

Accepted: 30/05/2021

Published: 02/07/2021

How to cite this article: Amado C, Silva Leal M, Neto P, Ferreira G. Subserous type of eosinophilic colitis: a rare disease. EJCRIM 2021;8: doi:10.12890/2021_002671.

Conflicts of Interests: The authors declare there are no competing interests.

This article is licensed under a Commons Attribution Non-Commercial 4.0 License

\section{ABSTRACT}

Eosinophilic colitis is a rare heterogeneous inflammatory disorder. The pathogenesis is not well understood even though it seems to be multifactorial, with hypersensitivity as a major contributor. The clinical presentation depends on the eosinophilic infiltration of different sections within the gastrointestinal tract. Diagnosis is based on the presence of peripheral eosinophilia and histopathological evidence of colon wall eosinophilic infiltration.

The authors present the case of a woman with a predominantly subserous pattern of eosinophilic colitis potentially triggered by intake of an Ulmus rubra-rich product.

\section{LEARNING POINTS}

- Subserous eosinophilic colitis (EC) is a rare inflammatory gastrointestinal disease that should be suspected when peripheral eosinophilia is accompanied by eosinophil-rich ascites and confirmed by the presence of eosinophilic infiltration of the colon wall.

- Although the aetiology of EC is not fully understood, a combination of genetic predisposition, dysbiosis and environmental factors (e.g., ingested allergens) likely contribute to it.

- The subserous type has the best prognosis and responds well to corticosteroids, with fewer relapses than other types.

\section{KEYWORDS}

Eosinophilic colitis, ascites, serosa, Ulmus rubra supplement

\section{INTRODUCTION}

Eosinophilic colitis (EC) is a rare inflammatory disorder that involves different gastrointestinal layers with different associated symptomatology ${ }^{[1,2]}$. The subserous type is the rarest presentation and is characterized by peripheral eosinophilia and eosinophilic ascites $^{[2,3]}$. Although difficult to estimate, the incidence of EC may be higher than previously thought ${ }^{[1]}$ with a greater prevalence in young adult women ${ }^{[2]}$.

The diagnosis is suspected when peripheral eosinophilia is accompanied by eosinophil-rich ascites (up to 95\% of cases), and confirmed by the presence of eosinophilic infiltration of the colon wall ${ }^{[1,3]}$. Crypt abscesses and lymphonodular hyperplasia may also be evident. Other causes of colonic eosinophilia should be ruled out ${ }^{[2]}$. The progression of disease in EC is variable: the subserous type has the best prognosis and responds well to corticosteroids, with fewer relapses than other types ${ }^{[2,3]}$. 


\section{CASE DESCRIPTION}

A 55-year-old Ukrainian woman with a medical history of arterial hypertension, dyslipidaemia, allergic rhinitis and penicillin allergy presented to the emergency room. She complained of a 7-day history of moderate and diffuse abdominal pain, nausea, diarrhoea and progressive abdominal distension. She denied fever, myalgia or arthralgia, night sweats or weight loss, recent alteration of her diet, new prescribed medications or recent travel. About 1 week before the beginning of symptoms, she took an Ulmus rubra-based supplement. The patient reported a similar episode 1 year previously, however, the aetiology was never clarified despite investigation. On examination, the abdomen was distended but soft, with a shifting dullness on percussion.

Laboratory examination revealed a high eosinophilic count at $8.73 \times 10 / \mathrm{ml}$ and no immature myeloid precursors, in an otherwise normal full blood count. Serum electrolytes, liver and renal function tests, coagulation screening and inflammatory markers were normal. Abdominal ultrasound demonstrated moderate ascites with no signs of portal hypertension, liver or renal disease. Diagnostic paracentesis was moderately cellular (7911 cells) with $87 \%$ eosinophils, sterile and negative for malignant cells, suggesting eosinophilic ascites.

The patient was hospitalized and the aetiological study was continued. Repeated blood analysis showed maintained marked eosinophilia, up to $14.2 \times 10 /$. Electrophoresis and immunoglobulin levels (including IgE) were normal. Serologies for HIV, HBV, HVC, Epstein-Barr virus and cytomegalovirus were negative. Stool analysis ruled out parasitic, viral and bacterial infection (including Helicobacter pylori), in addition to negative ELISA tests for Toxocara and Strongyloides. The interferon gamma release assay test was negative. Tryptase levels were slightly elevated and bone marrow aspiration and biopsy showed a marked increase in mature eosinophils without blasts. A thoracic-abdominopelvic CT scan was carried out with no significant findings other than moderate ascites. Upper endoscopy and colonoscopy demonstrated mild gastric and duodenum erythema and histology of biopsy samples revealed marked inflammatory eosinophilic infiltration in the lamina propria of the distal ileus and colon (Fig. 1). Echocardiographic findings were normal. The findings confirmed a diagnosis of subserous EC.

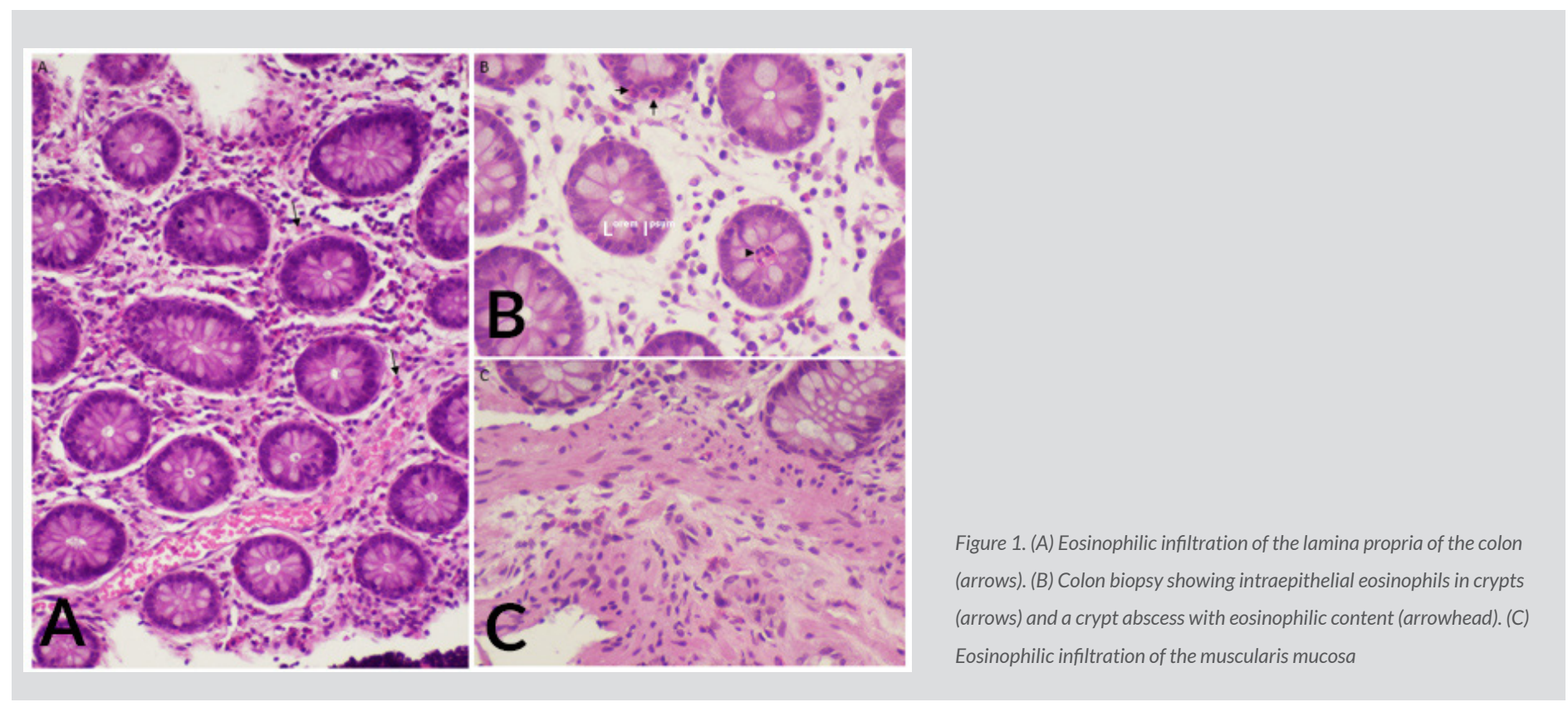

Based on the diagnostic criteria of Talley et al., namely, the presence of gastrointestinal symptoms, eosinophilic infiltration of the gastrointestinal tract on biopsies and no evidence of parasitic or extra-intestinal disease ${ }^{[6]}$, the diagnosis of eosinophilic colitis was established. The accurate exclusion of other possible conditions was very important because of their different treatments and prognosis.

The patient started systemic steroids with prednisolone $30 \mathrm{mg} /$ day with evident clinical and analytical improvement. After 2 weeks on steroids, symptoms had resolved, the full blood count was normal and the abdominal ultrasound showed no ascites. Corticosteroid tapering was started and the patient had maintained the response at the 2-month follow-up.

\section{DISCUSSION}

EC is a rare disease included in the group of eosinophilic gastrointestinal disorders and is characterized by peripheral eosinophilia and eosinophilic infiltration of the colon wall ${ }^{[2]}$. Unlike the presented case, it more frequently involves the mucosal and muscular layers ${ }^{[4]}$. Serosa 
involvement is typically associated with ascites, in which eosinophils are the predominant cell type in up to $95 \%$ of cases ${ }^{[2,3]}$.

In the presented case, the presence of gastrointestinal symptoms, peripheral eosinophilia and eosinophilic ascites suggested subserous EC, which was supported further by documentation of eosinophilic infiltration of the colon wall and the fast clinical/analytical response to steroid therapy.

There is much speculation regarding EC aetiology and it is thought that atopic conditions are the major contributor to its development ${ }^{[2,5]}$. In some series, about $50 \%$ of the patients had a history of allergies, so hypersensitivity to allergens resulting in immunodysregulation has been proposed as a pathogenic mechanism ${ }^{[6,7]}$. Taking into account the patient's atopy history and temporal causality, the authors believe there is a possible relationship with Ulmus rubra intake in a non-IgE-mediated hypersensitivity. A combination of genetic predisposition, dysbiosis and environmental factors (e.g., ingested allergens) is likely to contribute to EC ${ }^{[2,7]}$.

Eosinophilic colitis remains a rare entity currently lacking codified guidelines for diagnosis and treatment. In particular, well-defined threshold values for the diagnosis of EC on histological findings are still lacking.

Further studies are needed to better determine the underlying pathogenesis of this complex disorder, define and validate specific diagnostic criteria, compare the efficacy and safety profiles of the various available treatments and, finally, find a surrogate marker of disease relapse.

\section{REFERENCES}

\footnotetext{
Agrawal S, Vohra S, Rawat S, Kashyap V. Eosinophilic ascites: a diagnostic and therapeutic challenge. World J Gastrointest Surg 2016;8(9):656-659.

Impellizzeri G, Marasco G, Eusebio LH, Salfi N, Bazzoli F, Magari RM. Eosinophilic colitis: a clinical review. Dig Liver Dis 2019;51(6):769-773.

Shih H-M, Bair M-J, Chen H-L, Lin I-T. Eosinophilic gastroenteritis: brief review. Acta Gastroenterol Belg 2016;79(2):239-244.

Sunkara T, Rawla P, Yarlagadda KS, Gaduputi V. Eosinophilic gastroenteritis: diagnosis and clinical perspectives. Clin Exp Gastroenterol 2019;12:239-253.

Egan M, Furuta GT. Eosinophilic gastrointestinal diseases beyond eosinophilic esophagitis. Ann Allergy Asthma Immunol 2018;121(2):162-167.

Talley NJ, Shorter RG, Phillips SF, Zinsmeister AR. Eosinophilic gastroenteritis: a clinicopathological study of patients with disease of mucosa, muscle layer, and subserosal tissues. Gut 1990;31:54-58.

7. MingMing Z, YanQing L. Eosinophilic gastroenteritis: a state-of-the-art review. J Gastroenterol Hepatol 2017;32(1):64-72.
} 